\title{
Rapid Near-Field Antenna Testing Via Arrays of Modulated Scattering Probes
}

\author{
JEAN-CHARLES BOLOMEY, BARRY J. COWN, MEMBER, IEEE, GEORGES FINE, LUIS JOFRE, MEMBER, IEEE, \\ MASOUD MOSTAFAVI, MEMBER, IEEE, DOMINIQUE PICARD, JOHN P. ESTRADA, STUDENT MEMBER, IEEE, \\ PAUL G. FRIEDERICH, MEMBER, IEEE, AND FRED L. CAIN, SENIOR MEMBER, IEEE
}

\begin{abstract}
Key results are summarized of efforts to reduce significantly the near-field measurement time by utilizing one- or two-dimensional arrays of modulated scattering probes in lieu of the single probe ordinarily used in conventional near-field measurement techniques. Results of analytical, numerical, and experimental investigations show that the modulated scattering technique (MST) employing arrays of hundreds or even thousand of modulated scattering probes can be used to map the complex near-field of antennas or scatterers in a few seconds or minutes. The results also strongly indicate that "classical" (nonmodulated) receiving/transmitting arrays can be adapted for rapid nearfield data collection. Major factors affecting the accuracy and speed of probe arrays for near-field measurement are delineated and discussed. Experimental results obtained via laboratory prototype MST systems are also presented and discussed.
\end{abstract}

\section{INTRODUCTION}

$\mathrm{T}$ HE KEY results of research and development efforts to reduce near-field scanning time significantly by utilizing arrays of modulated scatterring probes are presented and discussed in this paper. The work described herein is motivated by the fact that the measurement times associated with the conventional near-field technique employing mechanical positioning of a single probe and/or test antenna can become excessively long for electrically large or sophisticated antennas-telecommunication antennas, phased arrays, multibeam antennas, reconfigurable antennas-which have to be tested for several possible excitations or configurations. The long measurement times associated with conventional nearfield scanning have also been an impediment to the on-site testing of antennas installed on vehicles, aircraft, ships, or located at field sites.

Manuscript received October 28, 1987; revised January 28, 1988. This work was supported in part by the Direction des Recherches et Etudes Techniques, the European Space Agency, the Centre National d'Etudes Spatiales, and the Agence Nationale pour la Valorisation de la Recherche in France and in the U.S. by the Georgia Tech Research Institute and the U.S. Army Communication-Electronic Command.

J. C. Bolomey, G. Fine, and D. Picard are with the Groupe d'Electromagnétisme, Laboratoire des Signaux et Systémes, Ecole Supérieure d'Electricité LSS-SUPELEC, 91190 Plateau du Moulon, Gif-sur-Yvette, France.

L. Jofre was with the Groupe d'Electromagnétisme, LSS-SUPELEC, 91190 Plateau du Moulon, Gif-sur-Yvette, France. He is now with E.T.S Ingenieros de Telecomunicación, Apdo. 30002, 08080-Barcelona, Spain.

M. Mostafavi was with the Groupe d'Electromagnétisme, LSS-SUPELEC

91190 Plateau du Moulon, Gif-sur-Yvette, France. He is now with the Department of Electrical and Computer Engineering, University of Missouri, Columbia, MO 65211.

B. J. Cown, J. P. Estrada, P. G. Friederich, and F. L. Cain are with the Electronics and Computer Systems Laboratory, Georgia Tech Research Institute, Georgia Institute of Technology, Atlanta, GA 30332

IEEE Log Number 8821113.
TABLE I

Estimated MEASUREMENT TIMES

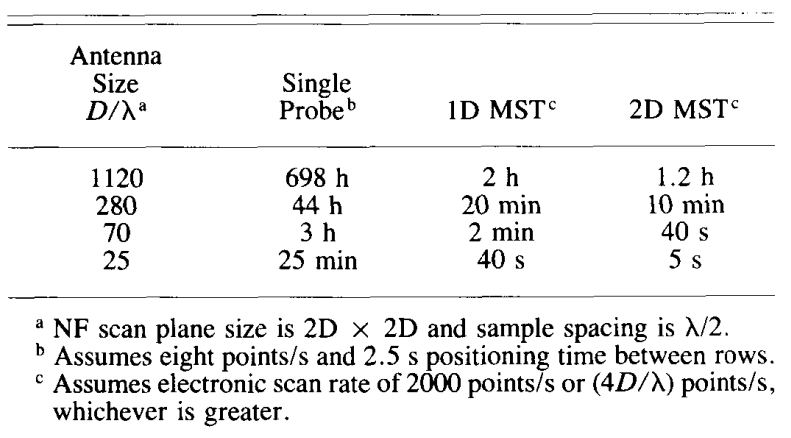

Dramatic reductions in measurement times for antennas as well as scatterers can be achieved via the use of the modulated scattering technique (MST), as evidenced by the estimated measurement times for conventional and MST-based near-field measurement systems presented in Table I [1]-[11]. The MST employs arrays comprised of hundreds or even thousands of electrically small scattering elements, each of whose scattering cross section is successively modulated by a low-frequency modulation signal to map the incident complex electric field of electrically large antennas or scatterers in a few seconds or minutes, depending on whether two-dimensional (2D) or onedimensional (1D) arrays are employed. MST arrays having large numbers of elements are typically more economical to build and deploy than "classical" (nonmodulated) receiving or transmitting arrays since MST arrays do not employ microwave switching networks, and hence only a lowfrequency multiplexer is needed. Some possible measurement configurations utilizing probe arrays are depicted in Fig. 1.

The use of probe arrays, whether MST or classical, introduces new sources of errors in the near-field measurement process. The potential difficulties most frequently cited against the use of probe arrays concern sensitivity and dynamic range limitations, interelement mutual coupling, and the effects of multiple interactions between the test antenna and the probe array. All of these factors may appear a priori to preclude the possibility of achieving high accuracy. However, the results of analytical, numerical, and experimental investigations show that all of the cited factors can be controlled and maintained at acceptable levels through careful design, fabrication and utilization of probe arrays and their associated electronic equipment. 


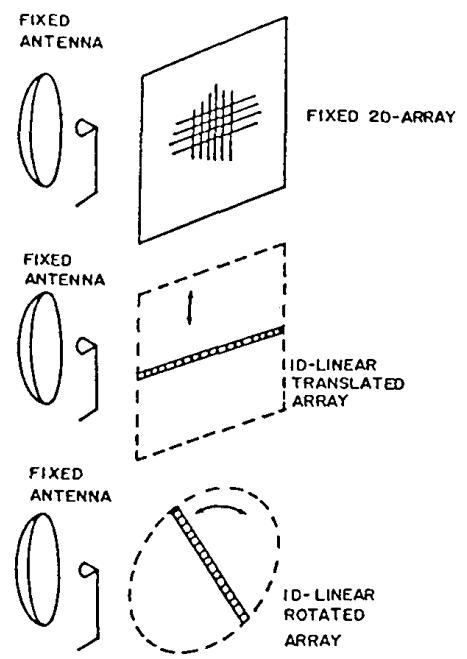

(a)

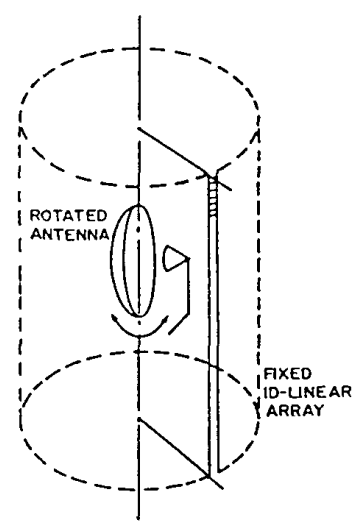

(b)

Fig. 1. Measurement setup for rapid near-field scanning via probe arrays. (a) Fixed antenna and movable test array. (b) Fixed test array and rotated antenna.

The remainder of this paper is organized as follows. The basic operating principles of MST-based measurement systems are briefly reviewed in Section II. Section III is devoted to a review of the effects of major factors affecting the accuracy and speed of MST probe arrays. This part includes previously published key results concerning dynamic range and interelement mutual coupling as well as new results on the effects of MST array-test antenna interactions. Experimental results obtained via laboratory prototype MST probe arrays are presented and discussed for horn and reflector antennas in Section IV. Concluding remarks are presented in Section V.

\section{Principle of MSt-Based Measurement Systems}

The MST is essentially a free-space perturbation technique. In that sense it is not the signal delivered by each probe array element to a receiver which is measured, but rather the field scattered by each probe array element. It can be shown via the reciprocity theorem that the signal resulting from the introduction of an electrically small electric field probe at a given measurement point is related to the component of the field $E e^{j \theta}$ at that point. In the monostatic case whereby the scattered signal is received by the antenna under test, the signal is proportional to $E^{2} e^{2 j \theta}$. In the bistatic case whereby the scattered signal is received by an auxiliary antenna, it is proportional to $E e^{j \theta}$. The respective advantages of both cases have already been discussed [3], [5]. Briefly speaking, monostatic arrangements suffer from possible $\pm \pi$ phase ambiguity and from a reduced dynamic range, both resulting from the quadratic relationship existing between the measured signal and the measured field component. However, monostatic setups can nevertheless provide relatively simple and compact solutions for some applications. Originally, the MST was developed to reduce the parasitic effect of the cable necessary to connect the probe to the receiver in conventional measurements [12], [13]. This parasitic effect was particularly disturbing for measurement of near-field aperture distribu- tions. The modulation was thus introduced to compensate for the low sensitivity and to improve discrimination against parasitic signals. In addition to these advantages, modulation makes it possible to record the field along the probe array by sequentially modulating each of the probes. Accordingly, one no longer needs a microwave multiplexer. Instead, only a lowfrequency (LF) or high-frequency (HF) multiplexer is necessary to apply the modulating signal successively to the probes. The modulation of the scattered field by single probes can be realized by several more or less sophisticated techniques which have been abundantly described [14]. It is worth noting that the concept of a modulated probe array for antenna measurements has not been introduced, to the authors' knowledge, before 1982 .

A typical MST experimental setup is depicted schematically in Fig. 2. A first coherent detection is achieved at the operating microwave frequency; a second one, at the modulation frequency, provides the real part and the imaginary part of the received signal. In such a setup, the measurement is not appreciably affected by nonmodulated signals that enter the signal channel, provided their level is less than the reference signal level or that they do not saturate the front end of the low noise amplifier, if one is used. These criteria can be satisfied in most experimental situations of practical interest.

\section{Accuracy and Speed Characteristics}

It is necessary to analyze and control major factors affecting accuracy, speed, and cost of MST systems. Engineering tradeoffs will probably have to be made to achieve specified levels of accuracy, speed, and cost for specific applications. Six basic factors that can have an impact on measurement system performances are as follows: 1) dynamic range, 2) interelement mutual coupling, 3) interactions between the MST array and test antenna, 4) parasitic signals (modulated and/or unmodulated), 5) dispersion of element scattering characteristics, and 6) probe correction. The results of numerical and 


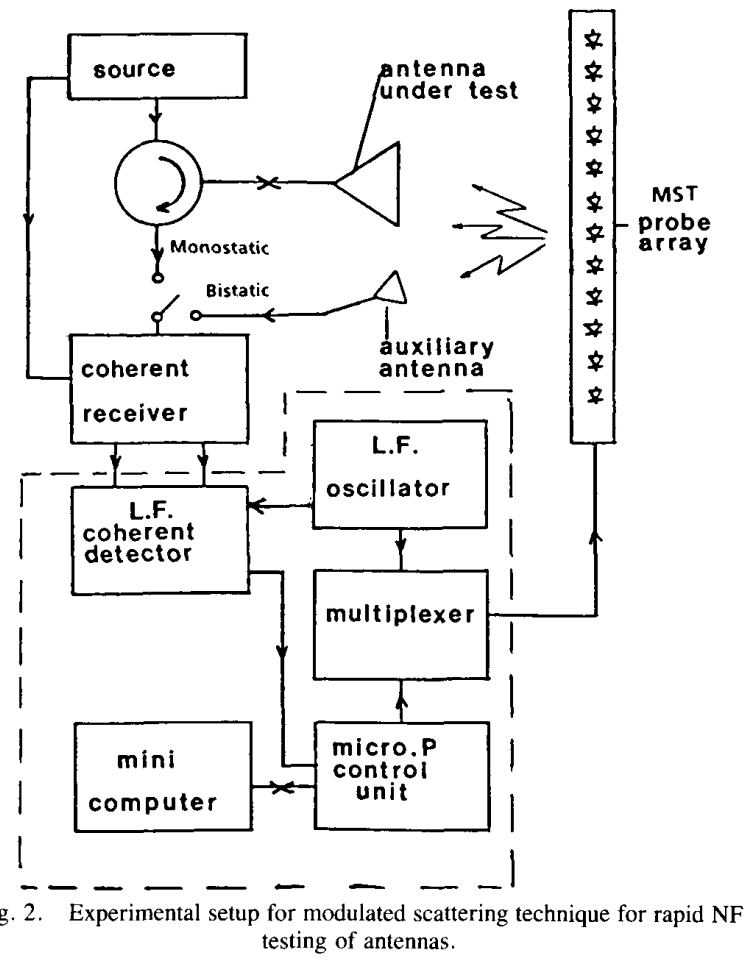

experimental studies show that the effects of the cited factors can be kept at acceptably low levels through careful design and implementation of the MST system. The effects of dynamic range limitations, interelement mutual coupling, and test antenna-MST array interaction are discussed further in following paragraphs. The influence of parasitic scattered signals from the near-field range is significantly reduced, due to the suppression of unmodulated signals by the homodyne receiver chain. The effects of element dispersion can be removed via a calibration measurement to determine the relative response characteristics of the MST elements to a known incident field. Probe correction for bistatic MST setups employing auxiliary antennas that are more directive than the test antenna can be accomplished with the aid of existing probe correction schemes combined with the calibration of the array element to correct for dispersion in element characteristics. Probe correction for the monostatic MST setups is more complex mathematically because the complex receiver voltage is proportional to the square of the near-field electric field, and conventional probe correction schemese are not directly applicable. Alternative probe correction schemes are under study for monostatic MST setups, as well as for bistatic MST setups employing auxiliary antennas having lower directivity than the test antenna [15], [16]. Fortunately, probe correction is a second-order effect for testing of electrically large antennas using MST arrays of short dipoles or waveguide elements having high symmetry in their scattering patterns.

\section{A. Effect of Near-Field Dynamic Range on Far-Field Accuracy}

The measurement dynamic range is related to the sensitivity and the rate of measurements. Limitation of the dynamic range obviously affects the far-field accuracy. The dynamic range $(\Delta D)^{2}$ and the rate of measurement $\tau$ can be defined in the following way [1], [17], [26]:

$$
\begin{gathered}
(\Delta D)^{2}=\frac{\Delta W_{s}}{W_{N}} \\
\tau=\frac{(\Delta D)^{2} F k T}{|\Delta \chi|^{2} W_{a}}
\end{gathered}
$$

where $\Delta W_{s}$ is the useful received power related to the input power $W_{a}$ of the test antenna according to the following relation:

$$
\Delta W_{s}=|\Delta \chi|^{2} W_{a} .
$$

$\Delta \chi$ can be considered as a transmission or reflection coefficient depending on whether a transmissive bistatic, reflective bistatic, or a monostatic setup is being used. It can be shown that $\Delta \mathrm{\chi}$ is a function of the antenna under test as well as geometrical properties (length, width) of the dipoles and the electrical properties (resistance, capacitance) of the diodes loading them [1], [3], [26]. The noise power $W_{N}$ is related to the noise factor $F$ of the receiver, the ambient temperature $T$, and the measurement rate $\tau$, according to the following approximate relation:

$$
W_{N} \simeq \frac{F k T}{\tau}
$$

where $k$ is Boltzmann's constant.

A numerical simulation was performed in spherical coordinates to examine the effects of near-field dynamic range limitations on far-field pattern accuracy. This simulation is based on the work done by Jensen et al. [18] and entails the following steps. First, a test antenna is chosen, and its exact near-field is calculated on a spherical surface. In this case, a paraboloidal reflector of $D / \lambda=30$ and $f / D=0.5$ is chosen as the test antenna, where $D$ is the diameter and $f$ is the focal length. Second, the exact near-field is perturbed by various errors [4] due to different sources, in this case the limitation of the dynamic range and the mutual coupling between probe elements. Third, the exact and the perturbed near fields are transformed to the far fields and the two results compared. The resulting deviations from exact values are then compared with accuracy requirements of the satellite antennas for 1980's [19]: copolarized main-beam directivity of $45 \pm 0.1 \mathrm{dBi}$, pointing direction of $\pm 0.01^{\circ}$, and 3-dB beamwidth of $0-0.75$ $\pm 0.01^{\circ}$; cross-polarized lobe of $-45 \mathrm{~dB} \pm 2 \mathrm{~dB}$ below peak of copolarized main beam. The near-field distribution of the chosen test antenna has a useful dynamic range of the order of $60 \mathrm{~dB}$. It is thus important to investigate the effect of limiting or truncating the near-field dynamic range on the far-field accuracy. This knowledge would also determine the rate of measurements. To simulate the limitation of the measurement dynamic range correctly, an added noise before the microwave synchronous detection is taken into account. Thus independently normally distributed noise using pseudorandom number generating subroutines is added to the real and imaginary parts of the near-field components to simulate the assumed dynamic 


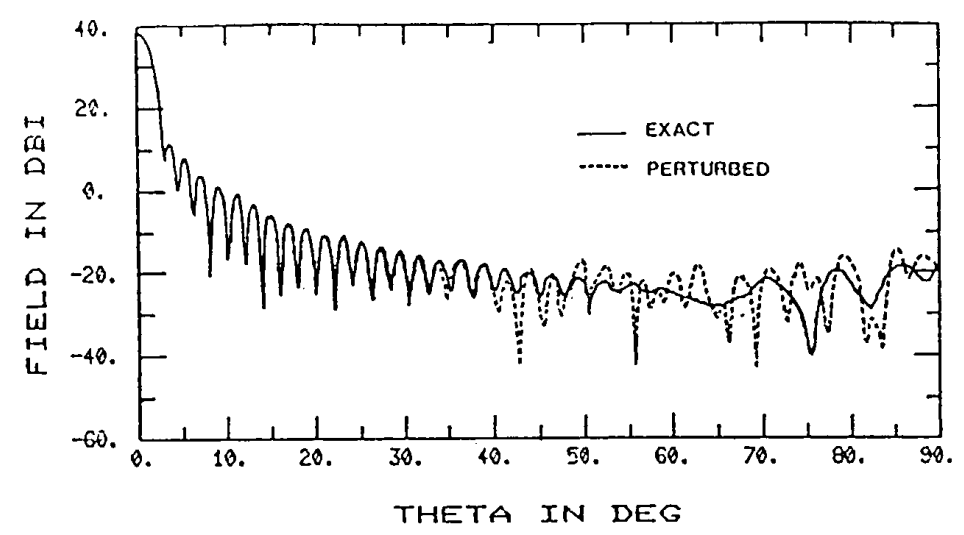

(a)

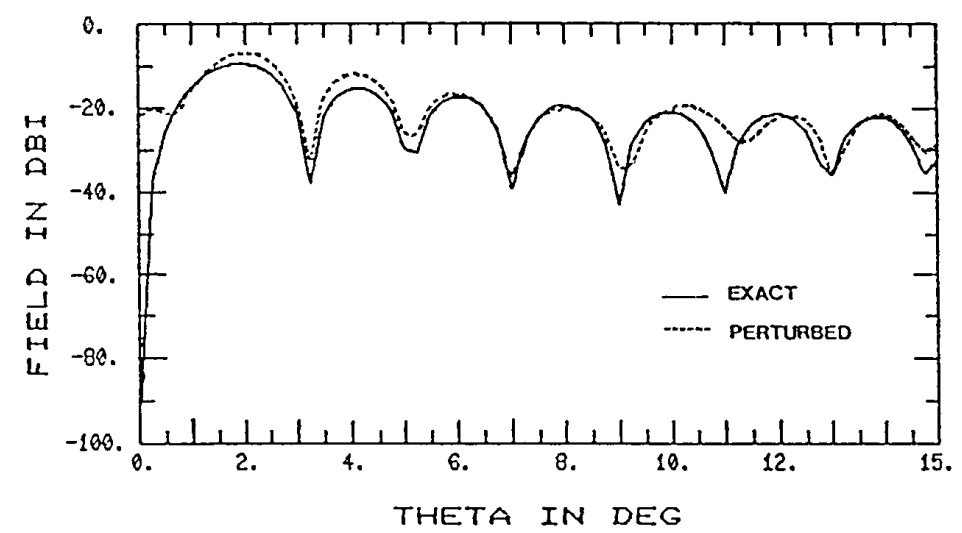

(b)

Fig. 3. Exact and perturbed cross-polar patterns. (a) For $\Phi=90^{\circ}$ for 40 -dB dynamic range. (b) For $\Phi=45^{\circ}$ for 50 -dB dynamic range.

range $(\Delta D)^{2}$. The three dynamic ranges considered are 1) $\left.(\Delta D)^{2}=60 \mathrm{~dB}, 2\right)(\Delta D)^{2}=50 \mathrm{~dB}$, and 3) $(\Delta D)^{2}=40 \mathrm{~dB}$.

Some typical results of the far-field characteristics are shown in Fig. 3. For the above three cases, the copolarized components are hardly affected in the range of $\theta$ that one is basically concerned with. For the worst case, i.e., the $(\Delta D)^{2}$ $=40 \mathrm{~dB}$ case, the far-field error for the copolarized peak is $0.036 \mathrm{dBi}$ which is smaller than the $0.1 \mathrm{dBi}$ tolerance previously specified. The cross-polarized deviation is still acceptable ( 2.7 versus 2 ) for the $(\Delta D)^{2}=50 \mathrm{~dB}$ case but considerably exceeds the $2-\mathrm{dB}$ tolerance for the $(\Delta D)^{2}=40 \mathrm{~dB}$ case. There is a noticeable degradation of the overall accuracy as the dynamic range is reduced from 60 to $40 \mathrm{~dB}$.

Simulations have also been performed for near-field data measured on a cylindrical surface for routine testing of monopulse antennas [5]. For these simulations, the aperture width in terms of wavelength was 40.0 . Plots of the far-field amplitude pattern versus angle are depicted in Fig. 4(a) and (b) for parabolic and parabolic-difference aperture distributions, respectively. Curves for dynamic ranges omitted from the plots were not graphically distinguishable from those plotted. These data indicate that even dynamic ranges of only $20 \mathrm{~dB}$ may be useful for some applications where the main beam and first few sidelobes of sum or difference patterns of highly directive antennas are of primary interest.

\section{B. Effect of Mutual Coupling on Far-Field Pattern Accuracy}

The mutual coupling between array elements is a source of error in the MST measurements. To calculate this coupling, the mutual admittance matrix of the array has to be evaluated. The model chosen is a linear array of dipoles with element separation $d=\lambda / 2$. A linear array of 11 dipoles with element length $l=\lambda / 6$, radius $a=\lambda / 200$ is considered to yield a sufficient degree of accuracy if one wishes to calculate the cumulative effect of coupling upon the central element. Investigation of the mutual admittance matrix of the abovementioned array has shown that the contribution of the elements beyond the fifth element is indeed negligible at the position of the central element. If the measurement distance is relatively large compared to the total length of 11 dipoles, a circular array can also be reasonably approximated by a linear array so far as coupling effects are concerned.

It can be shown [4], [17] by a multiport representation of the measurement setup that the voltage variation $\delta \rho(\Delta U 0)$ at the output terminal of the test antenna due to the modulation of the 


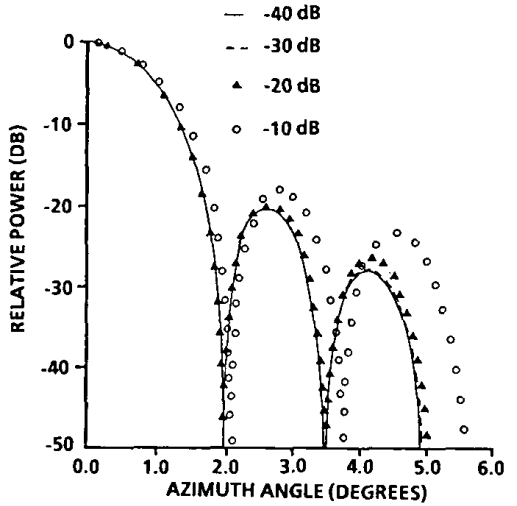

(a)

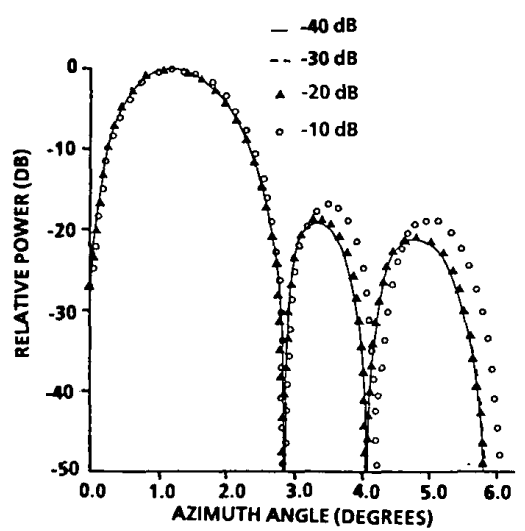

(b)

Fig. 4. Calculated antenna patterns. (a) For parabolic illumination function. (b) For parabolic difference illumination function for indicated near-field dynamic ranges.

$p$ th diode is given by

$$
\delta \rho\left(\Delta U_{0}\right)=\frac{-h^{2}}{I_{0}} \sum_{m=1}^{N} \sum_{n=1}^{N} E_{m} E_{n} \delta_{p} Y_{m n}^{\prime}
$$

where $Y_{m n}^{\prime}$ is the general admittance matrix with $\delta \rho Y_{m n}^{\prime}=$ $Y_{m n}^{\prime}$ ( $p$ th diode reverse biased) $-Y_{m n}^{\prime}$ ( $p$ th diode forward biased). $E_{m}$ and $E_{n}$ are the electric fields at the position of the $m$ th and $n$th dipole and parallel to it, $h$ is the effective length of each dipole, and $I_{0}$ is the current at the test antenna terminal. Note that the admittance matrix $\left[Y_{m n}^{\prime}\right.$ ] has to be found for each polarization orientation of the electric fields $E_{m}$ and $E_{n}$ corresponding to the orthogonal orientations of probe elements. Here, for example, the $\theta$ and $\Phi$ components of the field.

The method of moments (MM) is utilized to calculate the admittance matrix $\left[Y^{\prime}{ }_{m n}\right]$. For the above-specified dipole array, the coupling between two successive elements is calculated to be rather low, of the order of $-50 \mathrm{~dB}[4],[20]$, [3]. By increasing this value by $10 \mathrm{~dB}$ and $20 \mathrm{~dB}$, respectively, two other different cases with higher levels of coupling were simulated to take other possible sources of error into account. For example, introduction of different array elements, sub-

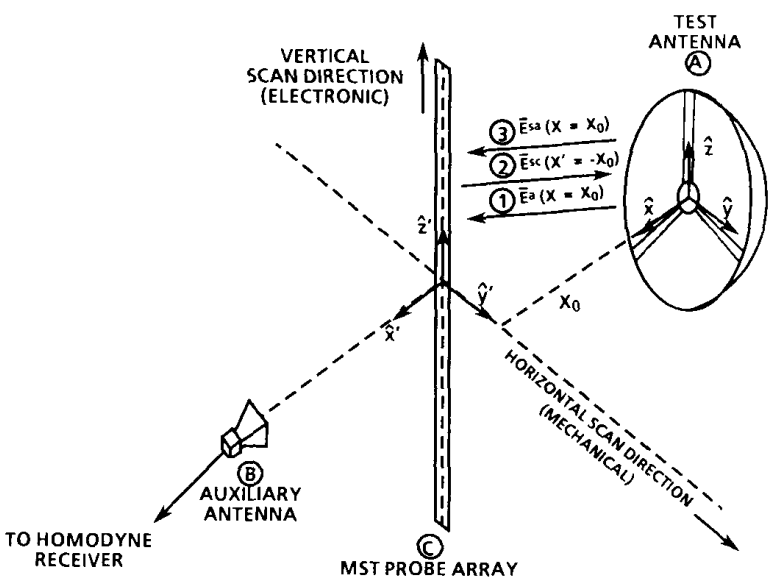

Fig. 5. Sketch illustrating first-order multiple scattering interaction for probe array and test antenna and showing pertinent geometrical parameters.

strate, modulation wires, etc. Three cases are thus treated in all. Measured values of mutual coupling between adjacent MST elements are about $40-45 \mathrm{~dB}$.

A numerical simulation similar to the previous one for the dynamic range effect was to run to evaluate the effect of mutual coupling. After the exact near field of the test antenna is perturbed by the coupling effect, the exact and the perturbed near fields are transformed to the far fields and the deviation between the two is calculated. Comparison of these valuesboth for the copolarized and cross-polarized cases-with the requirements previously mentioned show that the far-field errors due to the coupling effect of the dipole array fall within the given tolerances, if the cross-polarized level is not much below $40 \mathrm{~dB}$ of the copolarized peak.

\section{Effects of Test Antenna-MST Array Interactions}

Consideration of the basic near-field scattering and coupling mechanisms associated with MST probe arrays situated in the near field of an electrically large antenna shows that the dominant multiple scattering process for most situations is the one due to the fields designated as 1,2 , and 3 in Fig. 5. In particular, the electric field $\bar{E}^{a}$ of the antenna is incident on the probe array. The field $\bar{E}^{\text {sc }}$ scattered from the MST array is then incident on the test antenna, giving rise to the field $\bar{E}^{\text {sa }}$ scattered from the test antenna and which is incident on the MST array. The field $\bar{E}^{\text {sc }}$ scattered from the MST probe array is determined from the reflected field at the array surface via the application of forward and inverse fast Fourier transforms (FFT's) in accordance with well-known plane-wave spectrum (PWS) antenna analysis integrals [21], where the composite array substrate/MST element reflection coefficient is computed via a specialized moment method code for analyzing metallic elements on dielectric substrate [22]. The field $\bar{E}^{\text {sa }}$ scattered from the test antenna is comprised of two parts, the "antenna mode" scattered field and the "structural scattering mode" scattered field. The weighting coefficients for these two scattered field constituents are determined by invoking concepts from the theory of scattering from a loaded scatterer [23]-[25]. FFT's are then used to compute the field $\bar{E}^{\text {sa }}$ efficiently at the location of the MST 


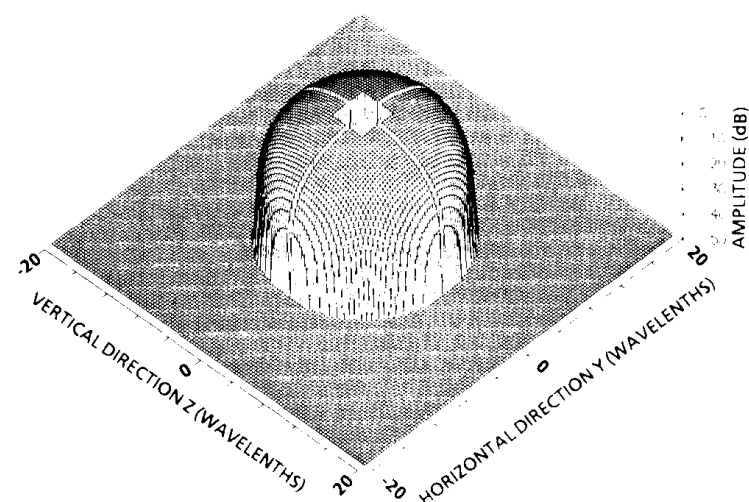

(a)

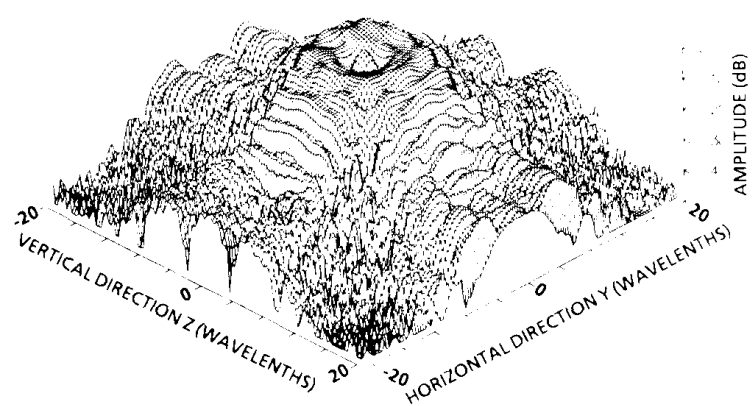

(b)

Fig. 6. Amplitude of electric field for $20-\lambda$ test antenna. (a) At antenna aperture. (b) On near-field scan plane at $X_{11}=10 \lambda$, where $\lambda$ is wavelength.

probe array, in accordance with the PWS near-field analysis technique previously cited. The computer program GTMINT and the analysis for analyzing multiple test antenna-MST probe arrays interactions are more fully described in [26].

The field $\bar{E}^{\text {sa }}$ is thus a perturbing field arising from firstorder multiple scattering of the unmodulated near-fields. The multiple scattering process can be continued to obtain the higher order field perturbations. However, the maximum value of the first-order perturbation field is found to be $<0.1$ $\bar{E}_{\max }^{a}$ and the second-order perturbation is $<0.01 \bar{E}_{\text {max }}^{a}$ for most measurement situations involving reasonably well matched test antennas measured with tall, narrow onedimensional MST arrays that are designed to have low backscatter. Accordingly, only the first-order perturbation, or “error" field $\bar{E}^{\text {sa }}$ is computed over the length of the MST probe array for each horizontal position of the array to obtain the two-dimensional map of the error field on the entire scan plane. The effect of the unwanted error field on the far-field pattern is obtained by superposition of the error field with the unperturbed or "true" field $\bar{E}^{a}$ on the scan plane and performing the near-field/far-field transformation.

Plots of relevant near-field and far-field electric ficld amplitudes are shown in Figs. 6-8 for a simulated measurement situation involving a $20 \lambda$ reflector antenna and a tall, narrow MST array scanned electronically along its vertical extent and moved mechanically along the horizontal direction. The MST array consists of short $(\lambda / 5)$ dipoles mounted on a thin dielectric substrate having a composite substrate/element

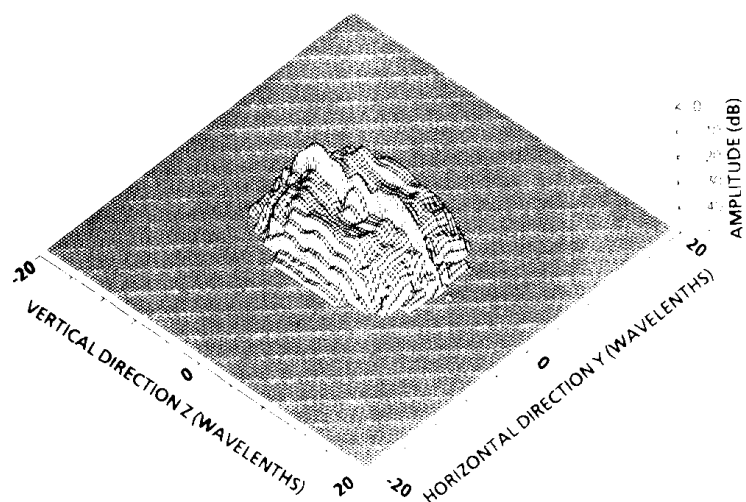

Fig. 7. Amplitude of near-field error field due to first-order multiple scattering between test antenna and MST probe array, for $X_{0}=10 \lambda$.

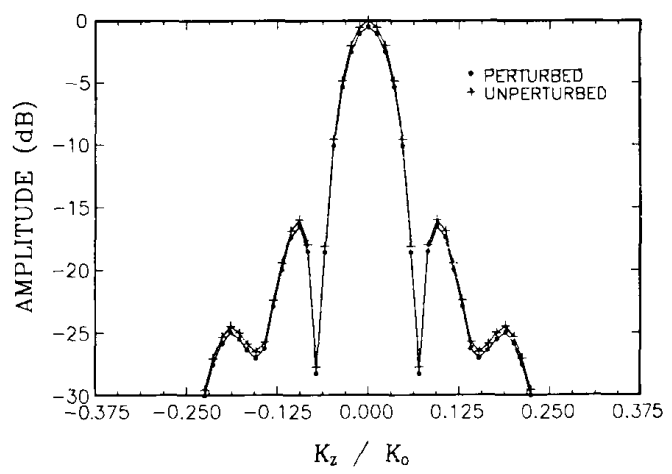

Fig. 8. Amplitude of far-field patterns for $20-\lambda$ reflector antenna

field reflection coefficient of $\Gamma=0.05$ and width $W=0.67 \lambda$. The overall voltage reflection coefficient arising from mismatch of the test antenna with its load is $\Gamma_{L}=0.1$. The antenna has four struts, and a $4 \lambda \times 4 \lambda$ square plate to simulate feed blockage. The aperture distribution produced by the feed is $\left(1-\rho^{2}\right)$. Inspection of the plots shown in Figs. 7 and 8 shows that the error field is marginally significant for this measurement situation. However, the magnitude of the crror field can be controlled and reduced further by careful design of the scattering properties of the MST array. For example, reducing the array reflection coefficient from 0.05 to 0.025 would reduce the boresight relative gain error from about $-0.5 \mathrm{~dB}$ to about $-0.22 \mathrm{~dB}$.

\section{Measurfments}

\section{A. MST Prototype Facilities and Test Antennas}

Experimental prototype MST probe arrays have been built and used to conduct feasibility and proof-of-concept tests involving horn and reflector antennas at both the Ecole Supérieure d'Electricité (SUPELEC) and Georgia Tech Research Institute (GTRI) [11], [26]. The measurement setup at SUPELEC pictured in Fig. 9 employs a one-dimensional array in the cylindrical scan geometry illustrated in Fig. 1(b), while the measurement setup at GTRI (not pictured) employs a onedimensional array in a planar scan geometry as per Fig. 1(a) except that the MST array is oriented vertically and moved 


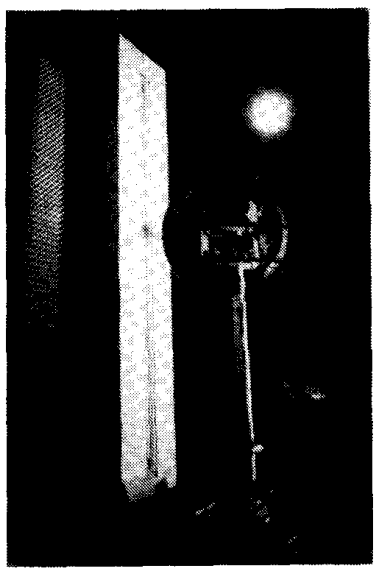

(a)

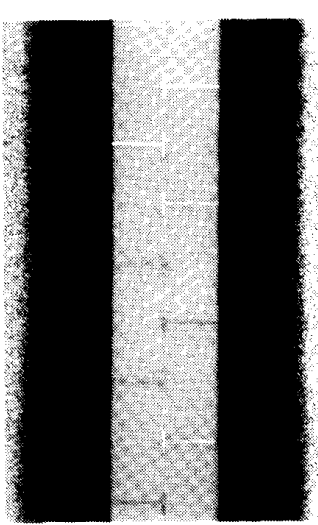

(b)
Fig. 9. MST setup for rapid cylindrical scanning. (a) From vantage point near location of test antenna. (b) Close-up view of MST dipole elements.

horizontally over the scan plane. Both prototype systems are designed to function in the $4-8-\mathrm{GHz}$ frequency range. The MST probe arrays at SUPELEC and GTRI each consist of 128 printed circuit dipoles mounted on thin (thickness $\simeq 30$ mils) glass epoxy substrate. For the MST array used at SUPELEC, the dipoles have length $=\lambda / 6$ and are spaced $\lambda / 2$ apart at 6.0 $\mathrm{GHz}$. For the MST array used at GTRI, the dipoles have length $\lambda / 5$ and are spaced $\lambda / 2$ apart at $8.0 \mathrm{GHz}$. Each diode is connected to the LF multiplexer that sequentially applies 25 $\mathrm{kHz}$ modulation via resistive or resistively coated lines. However, the two MST probe arrays are configured differently to investigate performance attributes of different probe array designs. The array used at SUPELEC has the dipole probes centered on the substrate and the flat side of the substrate is oriented toward the half-space containing the test antenna. The array used at GTRI has the dipole probes mounted at the edge of the substrate, and the edge is oriented toward the test antenna half-space.

The cylindrical MST setup at SUPELEC has been used to measure the complex near-field of two different horn antennas and a prime-focus fed parabolic reflector antenna. Most of the measurements have been conducted at frequencies from 5.8 to $6.1 \mathrm{GHz}$. The first horn tested is a nonuniform pyramidal horn constructed at SUPELEC and having an aperture of $5 \lambda \times 7 \lambda$ (at $6.0 \mathrm{GHz}$ ) in the $E$-plane and $H$-planes, respectively. The second horn is a $C$-band standard gain horn (Scientific Atlanta model 12-3.9) having $E$-plane and $H$-plane dimensions of about $3.2 \lambda \times 4.2 \lambda$ at the test frequency of $5.8 \mathrm{GHz}$. (This horn was provided and calibrated by the Technical University of Denmark.) The reflector antenna has $(F / D)$ ratio of 0.5 , and the diameter is 43.3 in, or about $22 \lambda$ at $6.0 \mathrm{GHz}$. For the MST measurements, the test antenna is rotated continuously while the MST array is scanned electronically along its length. The electronic scanning speed is selectable between 200 and 2500 points/s. Conventional measurements were made in the usual way by mechanically positioning a dielectrically filled $X$-band waveguide probe in the vertical direction for each angular position of the antenna. The test antenna was measured at the same sample points for both measurement techniques.

The planar MST setup at GTRI has been used to conduct 1) monostatic and bistatic MST measurements of a commercial (SA) standard gain horn of the same type as the one tested at SUPELEC and 2) bistatic MST measurements of a 48-in diameter prime-focus fed parabolic reflector antenna fed with an azimuth-only monopulse feed. These tests were conducted at $5.5 \mathrm{GHz}$. Accordingly, the horn aperture has $E$ - and $H$ plane dimensions of $3 \lambda \times 4 \lambda$, respectively, while the monopulse reflector antenna has a diameter of $\simeq 22.3 \lambda$. The $f / D$ ratio of the reflector is 0.5 . The near field measurements of the monopulse reflector antenna were conducted with the feed assembly electrically misaligned by a small amount to investigate the accuracy of MST probe array measurements vis $\grave{a}$ vis conventional measurement for characterizing the radiation patterns and tracking error curves for inadvertently misaligned antennas that may be encountered in practice. For the MST measurements, the MST array is mechanically scanned in the horizontal direction while the array is scanned electronically along its length in the vertical direction at a rate selectable between 100 and 2000 points/s. The auxiliary horn for the bistatic MST arrangements was a small horn located either below the test antenna or off to the side. Conventional measurements were also made in the usual way by moving a waveguide probe over the two-dimensional scan plane. The MST data and conventional data were typically measured at the same sample points.

\section{B. Experimental Results}

1) Pyramidal Horn Test Antennas: MST probe array and conventional results for the pyramidal horn test antennas are shown in Figs. 10-13. The data presented in Figs. 10-12 were obtained via the cylindrical scanning setup at SUPELEC, while the MST data presented in Fig. 13 were obtained via the planar scanning setup at GTRI. Close agreement is obtained between the MST probe array data and conventional data over the first 16-20 dB of dynamic range for the near-field and farfield plots. Note that the near-field measurement scanning times are indicated parenthetically on selected figure captions.

2) Reflector Antennas: MST and conventional results for the reflector antennas are shown in Figs. 14-17. Fig. 14 shows plots of the principal-plane patterns of the vertically polarized SUPELEC reflector antenna obtained via conventional and monostatic MST cylindrical scanning at SUPELEC. Numerical pattern predictions computed by the Centre National D'Etudes Spatiales (CNES) are also included. We note here that the measurement duration could be reduced to less than 1 min. by using a faster analog-to-digital converter. Similarly, Figs. 15 and 16 show azimuth patterns of the (misaligned) vertically polarized GTRI monopulse reflector antenna operating in the "difference" mode and the "sum" mode, respectively, obtained via the planar scanning setup at GTRI. Plots of the tracking error voltage versus azimuth angle are depicted in Fig. 17. The tracking voltage error $V(\Phi)$ was computed from the equation

$$
V(\Phi)=\left|\frac{E_{\Delta}(\Phi)}{E_{\Sigma}(\Phi)}\right| \cos \left[\beta_{\Delta}(\Phi)-\beta_{\Sigma}(\Phi)\right]
$$



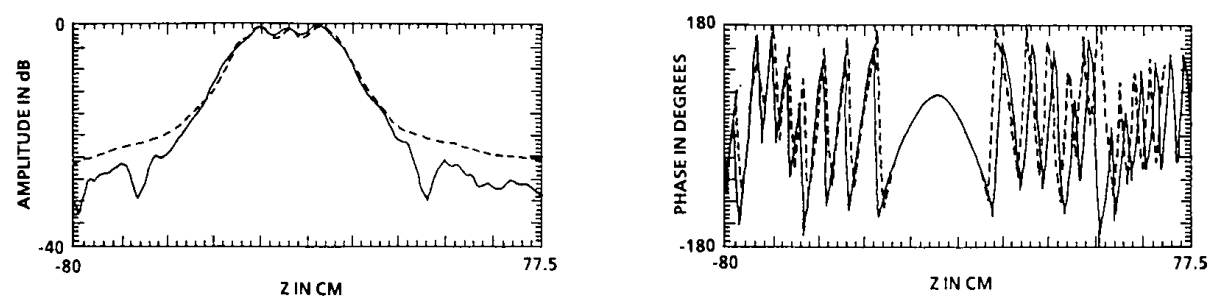

(a)
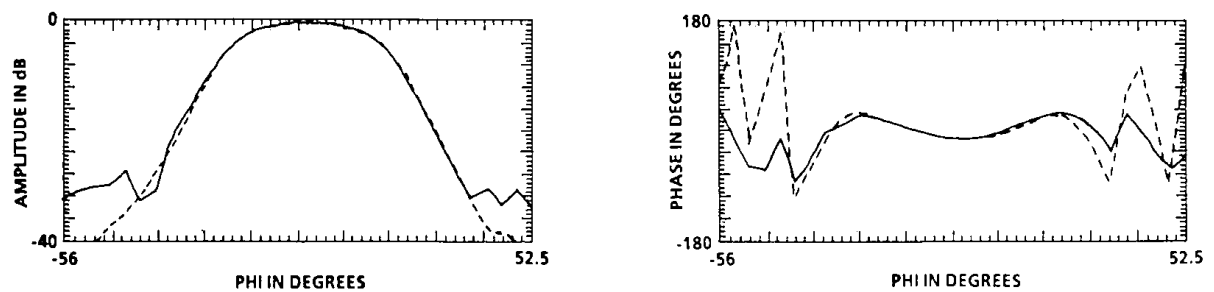

(b)

Fig. 10. Plots of amplitude and phase of near-field electric fields $E_{z}$ for SUPELEC horn. (a) Versus $z$. (b) Versus $\Phi$. _ - MST (45 s); ---- conventional $(50 \mathrm{~min})$.

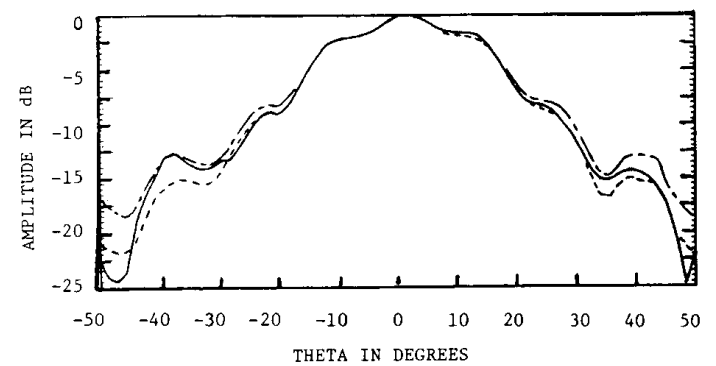

(a)

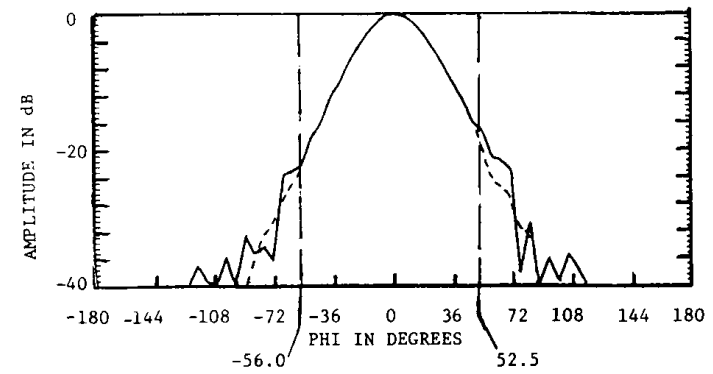

(b)

Fig. 11. Far-field patterns for $5 \lambda \times 7 \lambda$ SUPELEC horn computed from MST near-field data and from conventional near-field data. (a) $E$-plane pattern. (b) $H$-plane pattern. $\longrightarrow$ MST;,.,$\cdot \cdots \cdot$ conventional (ideal electric dipole probe correction); --.-- conventional (ideal magnetic dipole probe correction).

where

$E_{\Delta}(\Phi)$ complex far-field electric field for the difference mode,

$E_{\Sigma}(\Phi)$ complex far-field electric field for the sum mode,

$\beta_{\Delta}(\Phi)$ phase angle of the difference mode,

$\beta_{\Sigma}(\Phi)$ phase angle of the sum mode.

Close agreement between the MST probe array and conventional results as well as computed results is obtained over the

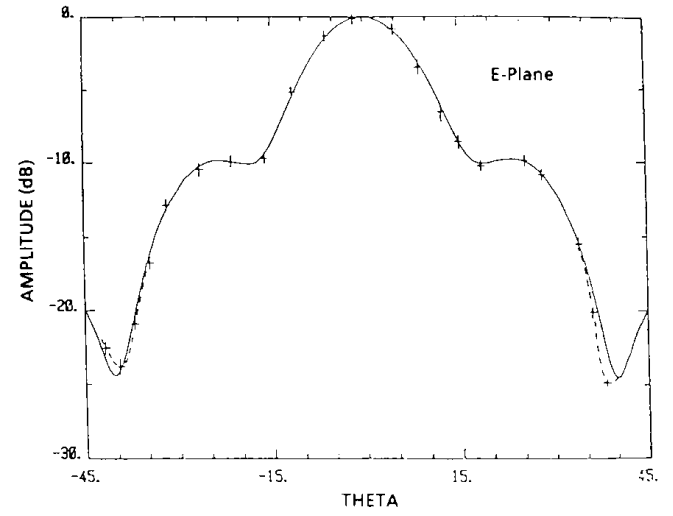

Fig. 12. Far-field pattern for SA standard gain horn measured at SUPELEC. - MST (45 s); -..- conventional (50 min).

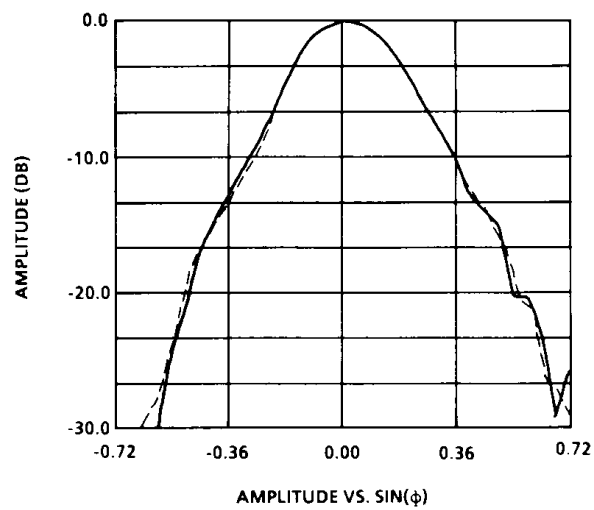

Fig. 13. Far-field aximuth patterns for SA standard gain horn measured at GTRI. - - MST (45 s); ---- conventional (40 min) 


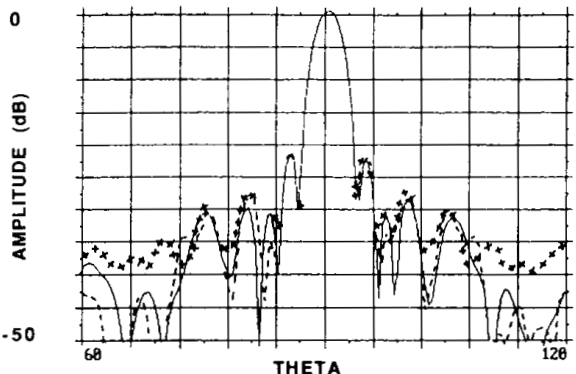

(a)

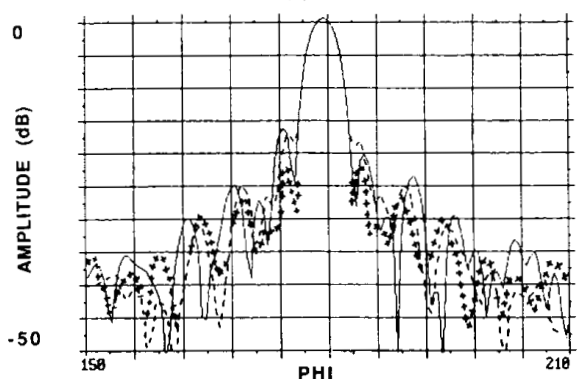

(b)

Fig. 14. Far-field patterns for 43.3-in-diameter reflector antenna measured at SUPELEC. (a) $E$-plane pattern. (b) $H$-plane pattern. — MST ( 7 min); ---- conventional $(70 \mathrm{~min}) ;++++$ numerical predictions.
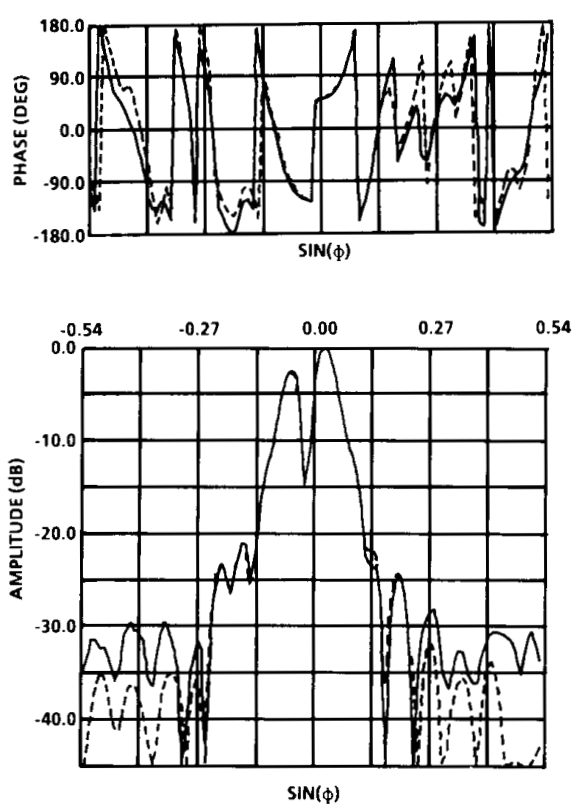

Fig. 15. Plots of far-field difference pattern for (misaligned) aximuth monopulse antenna measured at GTRI. - _ MST ( $3 \mathrm{~min}$ ); -... conventional (75 $\mathrm{min})$.

first 25-30 dB of dynamic range for the SUPELEC reflector elevation pattern (Fig. 14) and the GTRI monopulse reflector azimuth difference pattern (Fig. 15). Close agreement over the first $17-22 \mathrm{~dB}$ is obtained for the MST probe array and conventional results for the monopulse reflector azimuth sum pattern shown in Fig. 16 as well as for the azimuth pattern for
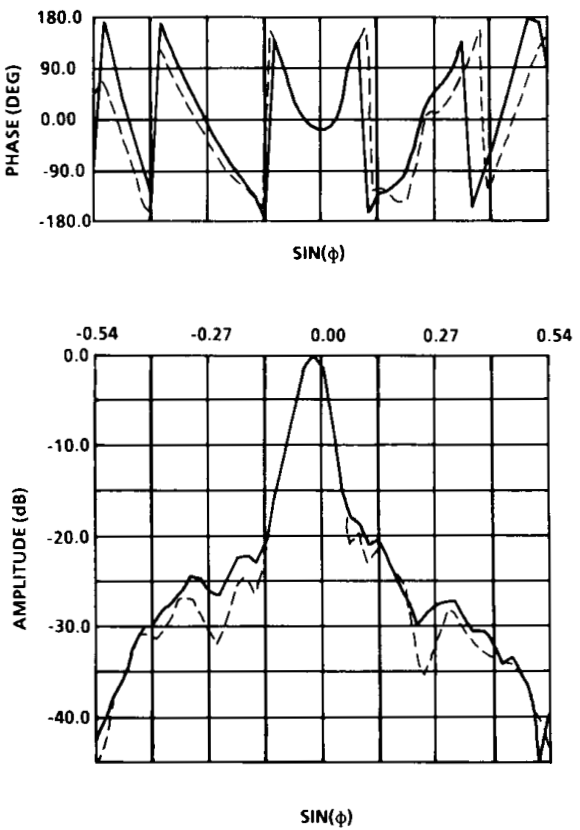

Fig. 16. Plots of far-field sum pattern for (misaligned) aximuth monopulse antenna measured at GTRI. - MST ( 3 min); -..- conventional (75 min).

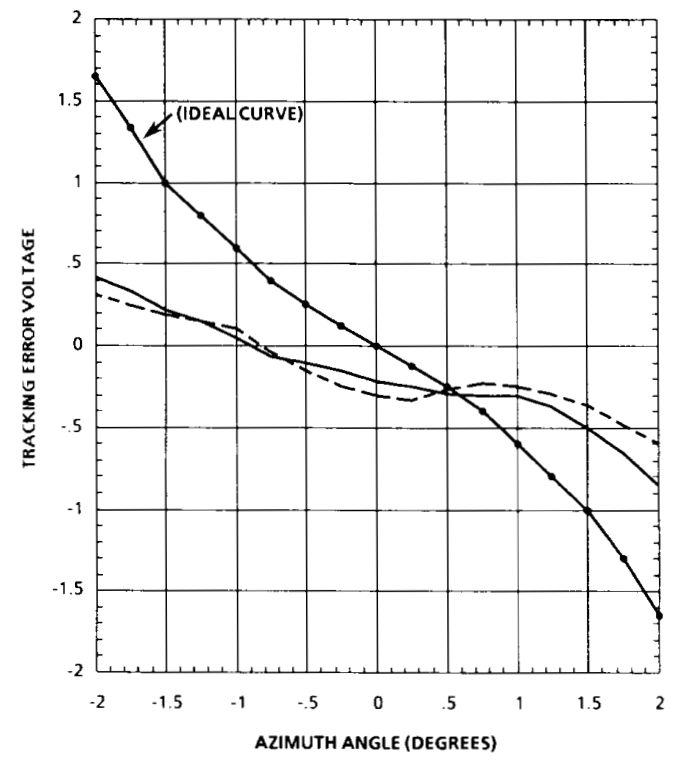

Fig. 17. Tracking error voltage versus azimuth angle for (misaligned) 48-in diameter monopulse antenna measured at GTRI. _- MST (3 min); -.-conventional (75 min).

the SUPELEC reflector antenna shown in Fig. 14 and most other pattern cuts.

The tracking error curves (Fig. 17) derived from MST probe array data and conventional data show reasonably close agreement over the entire tracking error voltage range. The zero-crossing points for the MST and conventional curves occur at $-0.91^{\circ}$ and $-0.80^{\circ}$, respectively, which yields a zero-crossing location error of $\simeq 2 \mathrm{mrad}$ for the two measurement techniques. 
The experimental results presented herein do not represent optimum or limiting results, and they can evidently be improved to increase accuracy and to include both broad-band frequency sweeping and cross-polarization measurement capabilities. Nevertheless, the results serve to demonstrate the potential of modulated probe arrays for rapid near-field scanning. Analytical and numerical studies indicate that the type of MST probe arrays described herein can be successfully used at millimeter wave frequencies and that useful RF measurement frequency bandwidths spanning at least one waveguide octave can be achieved. Experimental investigations of the millimeter wave performance and the useful RF measurement frequency bandwidths are subjects for future research and development efforts in this area.

\section{Concluding Remarks}

The slowness of current near-field measurements constitutes a significant impediment to the progress of near-field antenna testing technology. The MST is one possible solution which does not exclude classical approaches or any other innovative solution. However, the MST offers some attractive performance/cost attributes which render it particularly well suited for antenna testing applications as well as for general near-field applications where the speed/accuracy trade-offs are favorable for probe array scanning. Additional theoretical, numerical, and experimental studies have been initiated to define performance limits better and speed/accuracy/cost trade-offs among MST probe arrays, classical (nonmodulated) probe arrays, and conventional scanning. In closing, we note also that industrial nondestructive testing and biomedical diagnostic imaging are two important non-invasive testing methods which have suffered, until now, from the absence of suitable and rapid probing devices. The rather limited development of near-field microwave imaging and near-zone RCS applications may possibly be explained to a large extent by the lack of efficient means of rapidly acquiring and displaying data [27]. MST probe arrays as well as classical (nonmodulated) probe arrays should find additional applications in these areas in the near future.

\section{REFERENCES}

[1] J. Ch. Bolomey, "La methode de diffusion modulee, une approche au relevé des cartes de champs microonde en temps reél,' $L$ 'Onde Elect., vol. 62, pp. 73-78, May 1982

[2] G. Collignon, Y. Michel, F. Robin, J. Saint, and J. Ch. Bolomey, "Quick microwave field mapping for large antennas," Microwave J., pp. 129-132, Dec. 1982

[3] J. Ch. Bolomey, D. Picard, and M. Mostafavi, "Evaluation des cadences de mésure de champs proches au moyen de sondes modulées," Ann. Télécommun., vol. 40, no. 1-2, Jan.-Feb. 1985.

[4] M. Mostafavi, J. C. Bolomey, and D. Picard, "Far-field accuracy investigation using modulated scattering technique for fast near-field measurements," IEEE Trans. Antennas Propagat., vol. AP-33, no. 3, Mar. 1985.

[5] B. J. Cown, "Modulated scattering technique (MST) portable nearfield measurement system," Georgia Tech Res. Inst., Atlanta, Army Contract DAAB07-85-K-K515, Interim Tech. Rep., June 1985.

[6] B. J. Cown and J. C. Bolomey, "On the modulated scattering technique for rapid near-field measurements," in 1986 Nat. Radio Science Meeting Rec., June 9-13, 1986, Philadelphia, PA.

[7] J. C. Bolomey, G. Fine, M. Mostafavi, D. Picard, and B. J. Cown, "Reduction of near-field techniques duration," in Proc. 8th Annu. AMTA Symp., Sept. 1986.

[8] B. J. Cown et al., "Performance characteristics of the modulated scattering technique (MST) rapid near-field antenna measurement systems," in Proc. Int. Antenna Symp., Nov. 4-6, 1986, Nice, France.

[9] M. Mostafavi, D. Picard, G. Fine, J. C. Bolomey, and B. J. Cown, "Error considerations in cylindrical near-field scan using the modulated scattering technique," in 1987 IEEE AP-S Int. Symp. Digest, vol. 1, pp. 170-173.

[10] B. J. Cown et al. "Accuracy and speed characteristics of the bistatic MST for rapid near-field antenna measurements," in 1987 IEEE AP-S Int. Symp. Digest, vol. 1, pp. 174-178.

[11] D. Picard, "Mésure rapide de champs proche par la méthode de diffusion modulée," thése, Univ. Paris-Sud, France, July 1987.

[12] A. L. Cullen and J. C. Parr, "A new perturbation method for measuring microwave fields in free space," Proc. Inst. Elec. Eng., vol. 102, p. 836.

[13] J. H. Richmond, "A modulated scattering technique for measurement of field distribution," IRE Trans. Microwave Theory Tech., vol. MTT-3, (4) 1955(b)

[14] R. J. King, Microwave Homodyne Systems. London: Peter Peregrinus, 1978.

[15] G. Fine, "Contribution numérique et experimentale á la calibration de reseaux de sondes de mésure," thése en cours, Univ. Paris-Sud, Paris, France.

[16] B. J. Cown, Internal R \& D Project, Electronics and Computer Systems Lab., GTRI, Georgia Inst. Technol., Atlanta.

[17] J. C. Bolomey and D. Picard, "Etude du relevé rapide de champs proches par la méthode de diffusion modulée," Estec. Contract 5272/ 82/NL/GM (SC), Apr. 1983.

[18] F. Jensen, A. Frandsen, J. Aasted, and J. Hammer, "Experimental spherical near-field antenna test facility, MANUAL facility simulator FACSIM4,' ESA, Contract 3916/79/NL, June 1981.

[19] F. Jensen, "Experimental spherical near-field antenna test facility accuracy requirements evaluated from numerical simulations," ESA Final Rep. Contract 3916/79/NL/DG, vol. 2; Oct. 1981.

[20] M. Mostafavi, J. Ch. Bolomey, and D. Picard, "Etude du relevé rapide de champs proches par la méthode de diffusion modulée." Estec. Contract 5272/82/NL/GM (SC), Feb. 1984.

[21] D. M. Kerns, "Plane wave scattering matrix theory of antennas and antenna-antenna interaction: Formulation and application," J. Res. Nat. Bureau Standards, vol. 80B, no. 1, pp. 5-51, Jan. 1976.

[22] J. P. Montgomery, "Scattering by an infinite periodic array of thin conductors on a dielectric sheet," IEEE Trans. Antennas Propagat., vol. AP-23, pp. 70-75, Jan. 1975.

[23] D. Midgley, "A theory of receiving aerials applied to the re-radiation of an electromagnetic horn," Proc. Inst. Elec. Eng., vol. 108, pp. 645-650, Nov. 1961.

[24] R. B. Green, "The general theory of antenna scattering," Antenna Lab., Ohio State Univ., Rep. 1223-17, Nov. 1963.

[25] R. F. Harrigton, Field Computation by the Method of Moments. New York: Macmillan, 1968.

[26] B. J. Cown, "Modulated scattering technique (MST) portable nearfield measurement system," Georgia Tech Res. Inst., Army Contract DAAB07-85-K-K515, Final Tech. Rep., June 1988.

[27] B. J. Cown, C. E. Ryan, Jr., and J. J. H. Wang, "Potential near-field measurement techniques for determining near-zone and far-zone bistatic RCS," presented at the 1987 Symp. Antenna Measurement Tech. Assoc. (AMTA), Seattle, WA, Sep. 12-Oct. 1, 1987.

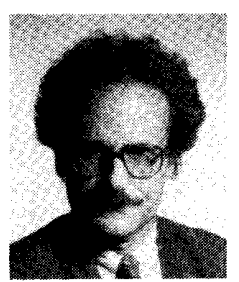

Jean-Charles Bolomey was born in Paris, France, in 1942. He received the electrical engineering degree from Ecole Supérieure d'Electricité, Gif/ Yvette, France, in 1963 and the Ph.D. degree from the University of Paris-XI (Orsay), in 1971.

He became Assistant Professor in 1974 and professor in 1976 at the University of Paris-XI (Orsay). His research activity conducted in the Groupe d'Electromagnétisme (CNRS-ESE) since that time, has been devoted to general scattering and diffraction, both numerical and experimental aspects. Since 1981, his main field of interest has been in near field imaging with a special emphasis to biomedical applications, antenna measurements and nondestructive testing.

Dr. Bolomey received the Blondel medal of the Sociéte des Electriciens et des Electroniciens in 1976, the General Ferié Prize de l'Académie des 
Sciences in 1984 and, with his group, the Microwave Prize of the European Conference in Nürnberg in 1983. From 1977 to 1982, he was President of Section 25 (ondes et signaux) of the Société des Eléctriciens et des Eléctroniciens. From 1978 to 1984 , he served as the French delegate to Commission B of the International Union of Radio Science.

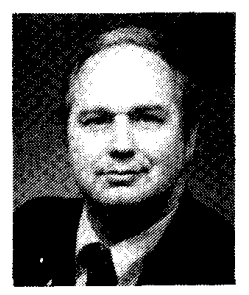

Barry J. Cown (M 75) was born in Rockmart, GA, on April 10, 1941. He received the B.S., M.S., and $\mathrm{Ph} . \mathrm{D}$. degrees in physics from the Georgia Institute of Technology, Atlanta, in 1971, 1975, and 1982, respectively

Since 1971 he has been a member of the research staff of the Georgia Tech Research Institute working on electromagnetic scattering analysis and measurement problems. He was a Visiting Research Scientist in the Groupe d'Electromagnétisme of the Laboratoire des Signaux et Systémes of the Ecole Supérieure d'Electricité in Gif-sur-Yvette, France, from September 1984 through September 1985 . His current interests are focused primarily on the development of probe arrays and generalized spectral analysis/MOM techniques to determine rapidly near-field radiation/scattering from antennas, equipment, and complex radar targets; near-field micro/millimeter wave imaging; and near-field antenna coupling.

Dr. Cown is a member of Sigma Pi Sigma and the AP and EMC Societies of IEEE, and he has served in various offices of the Atlanta Chapter of AP-S/ MTT-S, including Chairman.

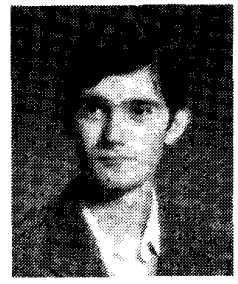

Georges Fine was born in Grenoble, France, in 1960. He received the engineering degree (Diplôme d'Ingénieur) in electrical engineering from Ecole Supérieure d'Electricité, Gif/Yvette, France, in 1983.

Since then he has been working on the antenna near-field measurements while preparing his doctoral thesis at the Electromagnetics Laboratory, Ecole Supérieure d'Electricité, Gif/Yvette, France.

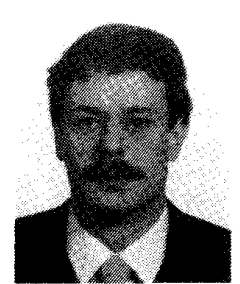

Luis Jofre ( $\mathrm{S}^{\prime} 79-\mathrm{M}^{\prime} 83$ ) was born in Mataró, Spain, in 1956. He received the Ingeniero and Doctor Ingeniero degrees in telecommunication engineering, both from the Universidad Politécnica de Cataluña (UPC), in 1978 and 1982, respectively. In 1978 he was a Research Assistant in the Electrophysics Department at UPC where he worked on analysis and near-field measurement of antennas. During 1981 he joined the Ecole Supérieure d'Electricité, where he was involved in microwave imaging techniques for biomedical applications. During 1986-1987 he was a Visiting Fulbright Scholar at Georgia Institute of Technology, Atlanta, working on antenna measurements and electromagnetic imaging. Since 1982 he has been an Associate Professor at the UPC where he is engaged in research in the areas of antennas and electromagnetic imaging, both in numerical and experimental aspects.

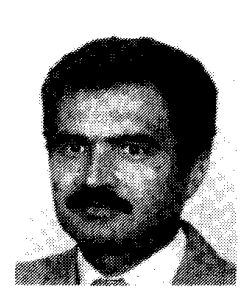

Masoud Mostafavi (M'84) was born in Isfahan, Iran, in 1946. He received the B.S., M.S., and Ph.D. degrees in electrical engineering from the University of Illinois, Urbana, in 1968, 1970, and 1975 , respectively.

From 1976 to 1978 he worked as a consultant. From 1978 to 1983, he was an Assistant Professor at the College of Meteorology and University Complex Engineering and Technology, Tehran, Iran. From 1983 to 1986 he worked as a Research Scientist at Ecole Superieure d'Eléctricité, Gif/ Yvette, France. Since 1986 he has been with the Department of Electrical and Computer Engineering, University of Missouri, Columbia, MO.

Dr. Mostafavi has been a member of Eta Kappa Nu, and the Société des Eléctriciens et des Eléctroniciens.

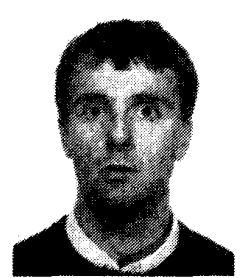

Dominique Picard was born in Chalons/Marne, France, in 1957. He received the engineering degree (Diplôme d'Ingénieur) in electrical engineering from Ecole Supérieure d'Electricité, Gif/ Yvette, France, in 1981 and the Ph.D. degree from the University of Paris-XI (Orsay), in 1987.

Since then he has been working on the antenna near-field measurements at the Electromagnetics Laboratory, Ecole Supérieure d’Electricité, Gif/ Yvette, France.

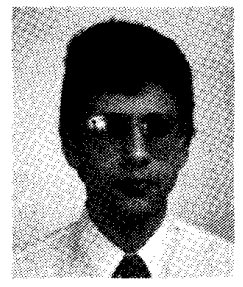

John P. Estrada (S'85-M'85-S'87) was born in Cocoa Beach, FL, on August 13, 1964. He received the B.S. degree in electrical engineering from Auburn University, Auburn, AL, in 1985 and is currently pursuing the M.S. degree in electrical engineering at Georgia Institute of Technology, Atlanta.

Upon graduation, he joined the Georgia Tech Research Institute as a Research Engineer. His topics of interest include antennas, and near-field measurement and analysis techniques.

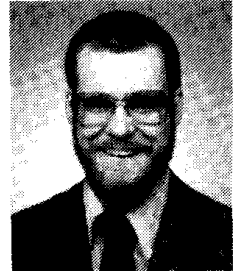

Paul G. Friederich (M'80) was born in North Carolina on April 26, 1957. He received the B.S degree in biomedical engineering from Vanderbilt University, Nashville, TN, in 1979 and the M.S. degree in electrical engineering from the Georgia Institute of Technology, Atlanta, in 1983

From 1980 to the present he has been a Research Engineer at the Georgia Tech Research Institute. He has worked in the areas of biomedical applications of microwaves and electrical properties of tissues at microwave frequencies. He has also been involved in microwave and millimeter wave measurements of ceramics at room and elevated temperatures, measurements of radar cross section (RCS) and RCS reduction properties of materials, and investigation of the effects of random damage to the performance of various microwave antenna types. He is currently involved in near-field antenna measurements and design of nearfield ranges.

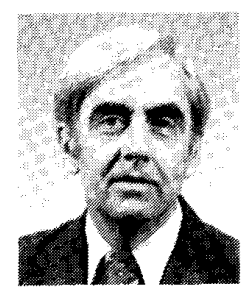

Fred L. Cain (S'62-M'66-SM'80) was born in Power, WV, on August 26, 1934. He received the B.S. and M.S. degrees in electrical engineering from the Georgia Institute of Technology, Atlanta, in 1963 and 1964, respectively.

He served as a communications consultant for the U.S. Eighth Army Signal Corps in Korea from 1957 to 1959 . Since 1964 , he has been with the Georgia Tech Research Institute at Georgia Tech where he has been involved with various aspects of applied research endeavors in the realm of electromagnetics. His major areas of research include antenna analysis and design, effects of environment on antenna performance, design of microwave components, near-field measurement techniques, electromagnetic compatibility, and beneficial applications of electromagnetic energy. he is currently a Principal Research Engineer and Director of the Electronics and Computer Systems Laboratory at Georgia Tech.

Mr. Cain's services within IEEE include the following: the various offices within the Atlanta Chapter of AP-S/MTT-S including President; member of COMAR (Committee on Man and Radiation); AP-S representative to COMAR; member of the 1974 IEEE International AP-S Symposium Publications Committee; and member of IEEE sponsored ANSI (American National Standards Institute) C95 Subcommittee of Radiation Hazards with Respect to Personnel. He is a member of the AP, MTT, and EMC Societies of IEEE and of Sigma Xi. 